DOI: $10.4301 / \mathrm{S} 1807-17752017000300003$

\title{
THE ADVANCED ANALYTICS JUMPSTART: DEFINITION, PROCESS MODEL, BEST PRACTICES
}

\section{Jeremy Rose \\ Mikael Berndtsson \\ Gunnar Mathiason}

University of Skövde, Skövde, Sweden

\section{Peter Larsson}

Advectas, Göteborg, Sweden

\begin{abstract}
Companies are encouraged by the big data trend to experiment with advanced analytics and many turn to specialist consultancies to help them get started where they lack the necessary competences. We investigate the program of one such consultancy, Advectas - in particular the advanced analytics Jumpstart. Using qualitative techniques including semi structured interviews and content analysis we investigate the nature and value of the Jumpstart concept through five cases in different companies. We provide a definition, a process model and a set of thirteen best practices derived from these experiences, and discuss the distinctive qualities of this approach.
\end{abstract}

Keywords: advanced analytics, big data, case study, best practice

Manuscript first received: 2017/Oct/20. Manuscript accepted: 2017/Nov/27

Address for correspondence:

Jeremy Rose, Professor of Informatics, University of Skövde, Skövde, Sweden.

E-mail: jeremy.rose@his.se

Mikael Berndtsson, Assistant Professor of Informatics, University of Skövde, Skövde, Sweden.

E-mail: mikael.berndtsson@his.se

Gunnar Mathiason, Assistant Professor in Computer Science, University of Skövde, Skövde, Sweden.

E-mail: gunnar.mathiason@his.se

Peter Larsson, Advectas, Göteborg, Sweden.

E-mail: Peter.Larsson@advectas.se 


\section{INTRODUCTION}

'A journey of a thousand miles begins with a single step'.

Lao, first century BC, the Tao Te Ching.

In the wake of the big data revolution, many organizations are experimenting with advanced analytics as a tool to help them stay competitive. Advanced analytics (AA) go beyond traditional business intelligence (BI) solutions based on performance indicators, dashboards and the querying of data warehouses to incorporate algorithmic techniques from machine learning, artificial intelligence, natural language processing and other computer science disciplines. Gartner define advanced analytics as follows: 'the autonomous or semi-autonomous examination of data or content using sophisticated techniques and tools, typically beyond those of traditional business intelligence (BI), to discover deeper insights, make predictions, or generate recommendations. AA techniques include those such as data/text mining, machine learning, pattern matching, forecasting, visualization, semantic analysis, sentiment analysis, network and cluster analysis, multivariate statistics, graph analysis, simulation, complex event processing, neural networks.' (Gartner IT, 2017). Though these techniques are established in some large international companies, and some specialist data and software companies, they represent a considerable learning challenge for the majority of companies.

AA projects are different from typical BI projects. Advanced analysis techniques are difficult to understand, even for the IT professionals in the companies, and represent black boxes for most decision makers. They may exploit external data sources (such as social media) as well as internal databases and unstructured data such as text and video as well as traditional structured data stored in transactional databases and data warehouse. This means that that the fusion of data is complicated by big data concerns (Kumar and Alencar 2016). They sometimes require dedicated hardware such as server clusters, and may use recent advances in database and software infrastructure (NoSQL databases, Hadoop, MapReduce). Many AA techniques depend on algorithmic techniques that can't easily be understood without specialist mathematical and statistical training - the new profession of "Data Scientist" denotes the specialists with these skills. They often derive patterns and insight autonomously from data (Saltz, 2015), rather than following routines in the software designed by humans. The approaches develop rapidly in their specialist research areas. AA may entail the computation of unfamiliar mathematical models (sometimes too complex for human comprehension) and the results can be presented in probability-based generalizations and new kinds of visualizations (such as scatter plots and confusion matrices). All these characteristics may challenge the understanding and decision-making tradition of business professionals. A further problem is that, although AA techniques can offer insights not available through conventional analysis (spreadsheets and dashboards, for example), they must carry some degree of trust before they are acted upon, and the analysis has no business value if there is no action resulting from it. Investments can be 'useless, even harmful, unless employees can incorporate that data into complex decision-making' (Shah, Horne, \& Capellá, 2012).

Since the majority of companies now see AA as valuable tools with the potential to improve efficiency and competitiveness, and to generate new business ideas, they must start somewhere to acquire AA competences. Consultancies with specialist analytics skills offer proof-of-concept solutions with high business-value, and are therefore key enablers for promoting widespread usage of AA. A common way of taking the first step is a consultancy-driven "Jumpstart". The Jumpstart (sometimes 
Kickstart) analogy likens the starting of a car with a dead battery (by attaching jump leads to an external power source) to getting the analytics motor of a company going through adding the energy of a specialist consultancy firm. The jumpstart/kickstart terminology is fairly broadly used in the IT consultancy industry, (such that the Google query 'jumpstart kickstart advanced analytics big data' returns 208,000+ hits); however the term here refers to a consultant-driven early-stage experimental project. Many IT consultancies offer these introductory services, though they don't always use the jumpstart terminology. After the project (or at some point in the future), the client company should be able to run their own AA program. Though there are a number of recommendations in the literature for becoming a data driven organization (Barton \& Court, 2012; Davenport, Harris, \& Morison, 2010; Marchand \& Peppard, 2013; Mazzei, McShea, \& Oakley, 2016; Vidgen, Shaw, \& Grant, 2017), most of this research is aimed at organizations experienced with data analytics. There is little research focused on inexperienced organizations in the start-up phase, and we found no investigations into the role of specialized consultancies in start-up. Moreover experience shows that 'hurdles on the path to effective analytics use are highest right at the start of adoption' (LaValle, Lesser, Shockley, Hopkins, \& Kruschwitz, 2011), so it should not be expected that a Jumpstart is necessarily easy or painless.

We studied the experience of a one such consultancy firm with a Jumpstart program. Advectas is an international company with 140 employees in four countries, with its headquarters in Sweden. They specialize in decision support, business intelligence, and, increasingly, advanced analytics. Our research objective is to investigate and document the Jumpstart concept to provide a reference point for future research, and to help specialist analytics consultancies to optimize their client experiences. We followed their Jumpstart project experiences with five client companies over a period of two years. The study investigates the nature and value of the Jumpstart process, and common enablers and obstacles in the joint consultancy/client projects. We used qualitative research methods, including interviews and content analysis. In the article, we describe the Advectas Jumpstart process, investigate what is known about getting started with advanced analytics in the scientific literature, report our research method and the five anonymized cases. Our case analysis leads to a definition of an advanced analytics Jumpstart, a process model, and thirteen best practices. We draw conclusions for practice and research.

\section{JUMPSTART AT ADVECTAS}

Jumpstart at Advectas is described to clients in terms of a process model, (in reality a modified version of CRISP-DM (Wirth \& Hipp, 2000)). It consists of two initial steps, followed by six steps in a cycle, Figure 1.

The two initial steps are:

- Business understanding: a two step investigation of (1) the targeted business problem, and (2) what the project should achieve.

- Data understanding: provides an overview of the client's available information situation.

The six step cycle consists of:

- Prio (short for prioritization): draws on the previous stages to identify a use case or scenario, the necessary data underpinning and candidate analytics techniques for the Jumpstart. Time and budget constraints, available staff and access to data should be agreed.

- Modelling: sets up the data and analytics techniques, defining variables and their relationships in relation to the chosen algorithmic procedures. 
- Evaluate: takes the modelling results back to the organization to check their validity and usefulness.

- Deploy pilot: provides a working prototype which runs the analytics program on a set of relevant data and provides meaningful results.

- Evaluate: takes the prototype results back to the organizations to investigate their usefulness.

- Deploy full scale: implements the prototype with live data and full function coding to a larger group in the client organization.

This process description provides a starting point for our study of five Jumpstart projects.

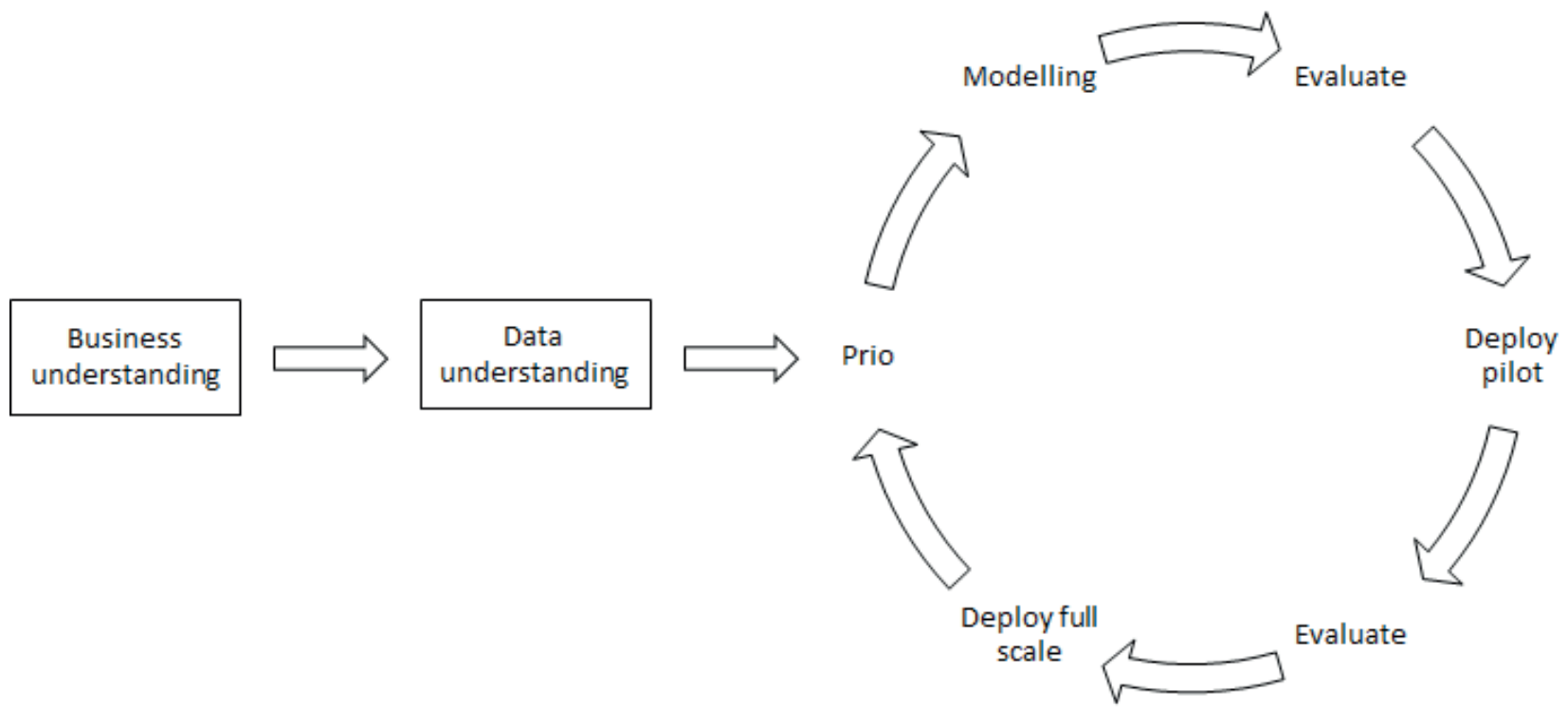

Figure 1. The Jumpstart method

\section{RESEARCH METHOD}

In this research we investigate Jumpstart practice for advanced analytics through a rich description of real life experience (Yin, 2013), making it appropriate to adopt an inductive case study approach. The study is exploratory in nature, aiming to formulate best practices by empirical exploration set within a theoretical context. Exploratory case studies typically address questions concerning the dynamics of a particular social situation (Eisenhardt \& Graebner, 2007), with the objective of developing early understandings. We use a structured case approach (Carroll \& Swatman, 2000) with an iterative research cycle: plan, collect data, analyze, reflect. We take care to frame our empirical findings with the enfolding literature. The study also involves elements of the multiple case study approach, since we analyze five smaller projects within the umbrella of the Advectas case. This typically provides a stronger empirical base than a single case study (Benbasat, Goldstein, \& Mead, 1987; Yin, 2013), and allows for cross-case comparisons to clarify whether an emergent finding is idiosyncratic, or replicated through several cases. Cause and effect patterns are retrieved by recursive cycling among the case data. 
We collected various kinds of data from multiple sources including document inspection, company web-sites, live interviews, semi-structured Skype interviews, company visits meetings and workshops over a two year period (see appendix A). Nine semi-structured interviews lasting 45-60 minutes with consultants and clients were conducted in total, and the interviews were recorded. We used early empirical findings, a case analysis (case A) and a literature study (see next section) to derive a research model (figure 2), since we were not able to find a suitable model in the literature. The research model structured a refined interview protocol and subsequent data collection.

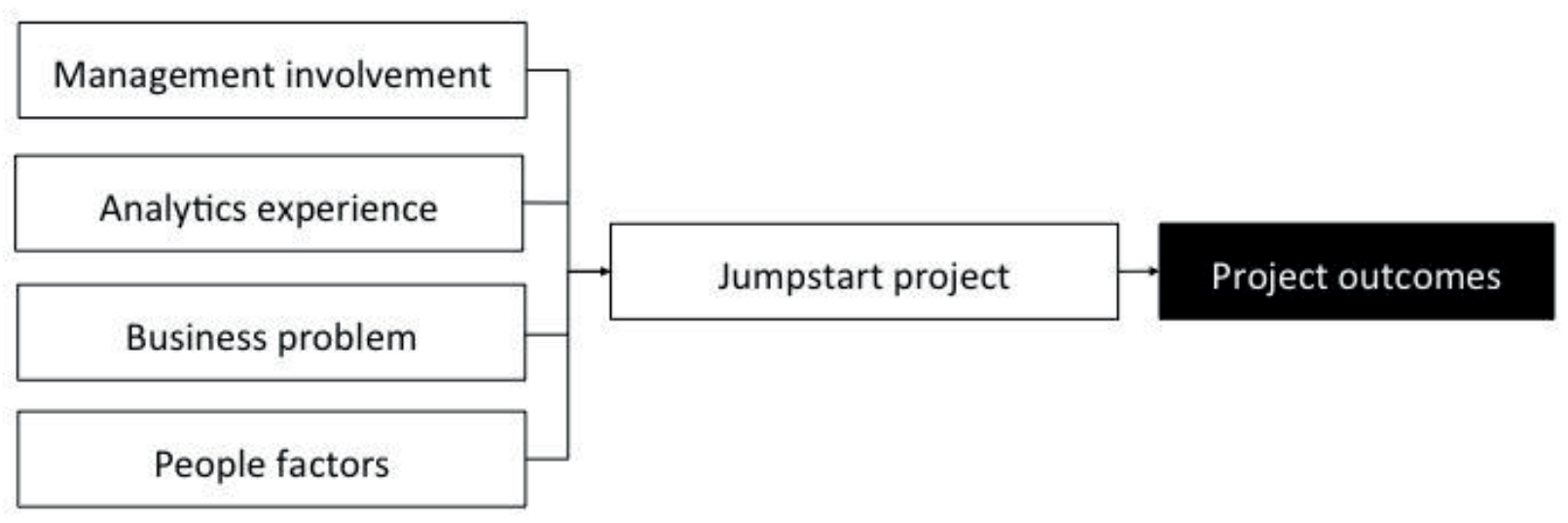

Figure 2. Research model for structuring of data collection

The model suggests that five main types of enablers influence the results of a Jumpstart:

- Management involvement: the degree, extent and type of involvement from more senior members of the client organization.

- Analytics experience: previous experience and competence with formal analysis of data, for example through a business intelligence project.

- Business problem: the nature and complexity of the business problem addressed.

- People factors: cultural, political and social influences (such as teamwork and trust) together with the prior knowledge and personal resources of the people concerned.

- The Jumpstart project: involving the conduct of the project itself, the methodology and tools used, and the systems, data and algorithmic techniques employed.

Beneficial outcomes can include business insights, information for decision-making, strengthening the client-consultant relationship, providing catalyst effects, and a variety of learning outcomes related to data and analytics.

The data analysis was conducted with content analysis (Berelson, 1952; Krippendorff, 2004). Content analysis yields 'a relatively systematic and comprehensive summary or overview of the dataset as a whole' (Wilkinson, 1997). In this case it operates by observing patterns of enablers and positive and negative outcomes in the Jumpstarts and categorizing them using a coding system. 
Categories can be elicited in a grounded way (as in our case) or can originate from an external source such as a theoretical model (Wilkinson, 1997). No quantitative measures for success were adopted; instead the analysis relied on the interviewees' assessment of the relationship between their project actions and perceived positive or negative outcomes. Three researchers coded the interviews from the original interview recordings, and the results were checked for consistency and concatenated, allowing analytic investigation by theme, by company or by frequency of appearance. A coding example is given in Appendix B. The emerging enablers were discussed and iteratively improved by comparison with the complete dataset, and then taken to a workshop at Advectas for refinement and validation. The best practices listed later represent concatenated normative versions of enablers identified during analysis.

\section{JUMPSTART ENABLERS}

There is a variety of research about business intelligence and big data projects in companies; here we summarise enablers that may be relevant to consultancy-driven AA jumpstarts. These insights form the starting point for data collection and analysis.

\section{Management Involvement}

The literature asserts that support from management is necessary for running any type of BIproject, including advanced analytic projects. A survey of executives by Brown and Gottlieb (2016) showed that ensuring involvement of senior management was the most significant reason for analytics success. In addition, respondents from successful analytic projects reported more often that they had received direct sponsorship from the CEO. Senior leaders and CEOs need to be actively involved in advanced analytic projects (Groves, Kayyali, Knott, \& Van Kuiken, 2013). For example, Mazzei et al. (2016) suggest that CEOs need to work on four things in order to keep their analytics project on the right track: i) actively manage $\mathrm{C}$-suite dynamics, ii) chose the right analytics leader, iii) challenge existing mental models, and iv) create an environment of rapid innovation. Involvement of senior management is also mandatory for any organization that has the ambition to establish an analytics enterprise-wide initiative, otherwise analytics initiative are likely to remain in siloes within the enterprise (Watson, 2016). Whereas theory suggests that top managers must be involved in enterprisewide analytics programs, it is much less explicit about start-up experiments, and seldom mentions the involvement of middle management.

\section{Analytics Experience}

Analytics are typically categorized as descriptive, predictive, and prescriptive (Davenport, 2013; Turban et al., 2015). Descriptive analytics are widespread even in smaller organizations and entail the investigation of what happened in the past through structured internal data held in the company's databases or migrated to a data warehouse solution built for the purpose. However, some degree of maturity in descriptive analytics, even at the level of data mining, does not guarantee success with advanced analytics. Predictive analytics use a variety of computational techniques to infer from historical data what may happen in the future and prescriptive analytics extend these insights to include decision options and their computed consequences. In addition to the algorithmdriven techniques of machine learning, statistical pattern recognition and natural language processing, advanced analytics may incorporate the V's of big data (Miele \& Shockley, 2013): volume (a large 
scale of data), velocity (streaming data) and variety (external and non-structured data such as text and video). Thus the competences required for advanced analytics are many and varied. Analytical competence development within an organization takes place in two categories: technical-oriented competence, and business-oriented competence (Davenport \& Patil, 2012). Technical-oriented competence development involves building up skills that resemble those of a data scientist. Businessoriented competence involves developing the ability to utilize analytics for business value. A recent success factor for wider adoption of BI and analytics (for instance within Tetra Pak, former winner of the Gartner BI Excellence Award) has been the introduction of a BI expert. A BI expert acts as a bridge between data scientists and IT-specialists on one side, and business specialists on the other (Ohlsson, 2017). A similar role, business translators, is suggested by Henke, Bughin, and Chui (2016). Maturity models (Lahrmann, Marx, Winter, \& Wortmann, 2011) theorize and assess the level of maturity, and an organization with better existing maturity at the start of its analytics Jumpstart is assumed to be more likely to succeed with it. LaValle, Hopkins, Lesser, Shockley, and Kruschwitz (2010) introduced a maturity model for analytics with three levels: aspirational, experienced, and transformed. For each level six factors are investigated: motive, functional proficiency, business challenges, key obstacles, data management, and analytics in action. The model was introduced to help organizations to assess their analytics maturity. The maturity model does not explicitly focus on progression of analytical competence within an organization. However, progression can to some degree be deduced from investigating the three factors: motive, business challenges, and key obstacles.

\section{The Business Problem}

Chen, Chiang, and Storey (2012) list five areas where AA are likely to have a big impact: e-commerce and market intelligence, e-government and politics 2.0, science and technology, smart health and well-being, and security and public safety. In practice the business needs of organizations vary considerably, and an early stage in adopting advanced analytics is simply demonstrating their value on a relevant business or organizational problem. Suitable problems explore data, for instance categorizations, predictions, associations, or prescriptions. The organization tends to deal with these problems in a time-consuming manual manner, or regard them as unsolvable. Since AA are normally data driven (Chen et al., 2012), it's common to select open problems and allow the analytics to automate the search for hidden patterns or trends in the data. Where patterns or trends appear interesting or unusual the next step is to develop interpretations or models that explain them (He, Zha, \& Li, 2013). However Rose and Lennerholt (2017) point out that this style of problem solving makes it hard to focus on a defined problem and to solve it by hypothesis testing. As organizations become more familiar with AA techniques, problem choice becomes simpler, speed to insight improves and use becomes more pervasive (Wixon, Yen, \& Relic, 2013).

\section{People Factors}

Working with advanced analytics requires that people use analytical tools to investigate and learn about a business problem, in order to improve decision-making. Thus 'analytical techniques and controlled experiments are tools for thinking' (Marchand \& Peppard, 2013). However it is the people who do the actual thinking and learning, and who make decisions. There are usually at least four categories of people involved in an advanced analytics project: data scientists, staff from the IT department, business people and management. The challenge is to get these diverse skill sets to work together to investigate a business problem. Managers in particular face many problems with advanced 
analytics projects: analytics force changes on the C-suite (Mazzei et al., 2016), many business people (including managers) are uncomfortable working with data (Marchand \& Peppard, 2013), managers don't understand or trust big data based models (Barton \& Court, 2012).

\section{The Jumpstart Project}

Researchers offer two conflicting models for getting started with AA. The high impact model suggests the start should be substantial, influential and pivotal for the whole organization's future: 'the challenge should be big, the model insightful and the business vision complete' (LaValle et al., 2011). The low cost model focuses on the opposite strategy: small-scale experiments through targeted prototyping for a quick win. According to (Franks, 2012) companies should 'define a few relatively simple analytics that won't take much time or data to run .... capture some of the data in a one-off fashion.... turn analytic professionals loose on the data.' Gust et al. (2017) describe an internal seed project in a Swiss company with some of the same characteristics as a Jumpstart. As for team configuration, emphasis should be put on a small mixed team that work well in a test and learn environment. Having a small group of people with mixed competences offers several advantages in terms of quality, speed and flexibility. The bigger the group is, the longer it may take to complete the project. In an ideal world, a Jumpstart project should mimic a project in a data-driven organization, where business people and data scientists continuously run experiments together in order to learn more about their business (Watson, 2016). This presents challenges both to conventional analytical processes (ask the IT department to prepare a report) and intuition-based decision-making. A method similar to CRISP-DM (Wirth \& Hipp, 2000) is often adopted, though some researchers combine this kind of approach with system development methods such as agile development (Larson \& Chang, 2016).

\section{FIVE JUMPSTARTS}

This section provides a short background to the five Jumpstarts that were conducted. Company names have been anonymized.

\section{Company A}

Company A operates transport services for freight and passengers between the major destinations of Europe. Advectas were invited to work with Company A's marketing and IT departments as a part of a wider vision to become data driven - a company which made decisions on the basis of data analysis. Advectas cooperated with the marketing department and operated as a specialized resource for the IT department, but the company's business intelligence group was not much involved. Four Advectas consultants participated and at least ten people from Company A, including relevant business managers. The project focused on business questions around the passenger loyalty program (what they buy, how often they travel, what the value of the program is, etc.). The project should develop a test environment and some models that should eventually help marketers to conduct much more sophisticated data analysis than their simple data queries and excel sheets currently enabled. Two workgroups with business managers and IT worked on what some eventual dashboards should look like look like and provided a requirements specification. As the project progressed, building the test environment turned out to involve: 
- porting data from source systems including the company's aging customer relationship management systems and their website usage analysis software,

- integrating it in a data lake based on Microsoft Azure Platform

- developing analysis models including some advanced analytic machine learning, primarily in the PowerBI tool, and Azure Machine Learning

- making the environment available to marketers to run their own custom analyses.

The first three objectives were achieved but there were many difficulties to solve along the way. The lack of a clear business (marketing) objective led to many different demands and foci for the project. "In the beginning, it was a bit loose ... exactly what data should we use ... [move the data] up in the cloud and then run Power BI ... and some machine learning" (Consultant 1). Later there were rapidly expanding requirements and ambitious expectations. There were many problems with the data, including quality problems, legal problems, and missing data when it came to modelling important problem variables. PowerBI turned out to have limitations in relation to the more advanced models and analytics. There were organizational problems as well, even with simple things like getting hold of necessary software licenses. The marketing department saw the project as an opportunity to bypass the IT department and get the systems they wanted built, but had little expertise in formulating what these systems should look like. At the same time they did not really trust the data or the results they got. Top management was involved in the launch of an enterprise-wide program for data driven decision making, in which the Jumpstart project served as one of the initial steps. However, the overall vision for data driven decision making had uneven commitment from managers and little buy in from staff. Both IT staff and marketers felt threatened by the different approach and new competences required - "[they] were very worried that the machine-learning part would continuously run live analysis and generate the same insights as they [the business people] did" (consultant 1). Advectas understandably focused on the technical development and it was unclear who was leading the necessary organizational change program - "the technical solution, is still rather straightforward and simple, ... but then the other things swelled up" (Consultant 1). The project did not continue to a production system but had important catalyst effects for Company A. They understood a clear need for better data quality and analysis competence and appointed a data quality specialist, a mathematician and a marketing analyst to fill these gaps.

\section{Company B}

Company B is a Scandinavian retail chain, with more than 200 shops in Sweden, Norway and Finland. The project had a budget of 100 consultant hours and involved two Advectas data analysts and three IT staff from Company B. The IT department was interested to find out what could be achieved with data analytics, but had no serious history with them. They hypothesized that sales might vary with the weather ("many opinions for or against [a potential pattern]" (Consultant 2)), though the business staff were not much involved in the project. However, Company B had no appropriate tools to investigate the hypothesis further on their own. The project focus was set to investigate correlations between historical sales data with location and publically available weather data. The analysts from Advectas settled eventually on a model using the random forest algorithm, and were able to illustrate several interesting correlations, through two deliveries. The final event was a presentation to which senior business managers were invited, which provoked some inspiring discussion and business suggestions. According to consultant 2, "everybody trusted the result ... there was no scepticism ... 
it was more constructive discussion". It is not known whether these suggestions were developed or implemented in the business. Advectas' competences were demonstrated and Company B intend to pursue analytics, but there was no follow up cooperation at the time of writing.

\section{Company $\mathrm{C}$}

Company $\mathrm{C}$ is a recycling company in Sweden. The Jumpstart arose from earlier contacts and projects in sister companies. Five Advectas staff were involved intermittently, and several managers, two in particular, were involved from Company $\mathrm{C}$. The production managers were more enthusiastic for the project than the operators, who were however happy to provide input, and the IT staff were only superficially involved. Company $\mathrm{C}$, wanted an explicit small scale proof of concept which would show that advanced analytics could make the business more effective by optimizing the smelting furnace operation. The furnace operates a continuous cycle which is expensive to run, and where small optimizations save a lot of money and increase production capacity significantly. However there are many parameters (temperature, gas, oxygen, impurities in the raw material, filters) in running the furnace, and various control systems, making it dependent on operator knowledge, skill and experience. Data is provided by 10-15 different sensors in the furnace + visual input. A lot of data was provided, but there were consistency and quality problems, different operation modes, and some encrypted data. According to consultant 3, "it became a jouney of a thousand miles to arrange all that information ... we had to put in lots of extra time [to fix data quality problems] .. several months of delay". The project was in the model development stage at the time of writing, with good potential to codify and automate the operational experience of the operators.

\section{Company D}

Company D operates a large fleet of tankers carrying cargoes of petrol, chemicals and liquefied gas all over the world. Since fuel oil is a major cost for Company D, they cooperated in a Jumpstart with Advectas designed to help them predict Worldscale rates 14 days in advance. Worldscale rates are used to calculate freight charges and based on several parameters such as bunker prices, port costs, and market rates. In the beginning, expectations were low - "the [business specialist] who worked with it [manual prediction] was very sceptical that a model would be able to guess prices 14 days in advance" (Consultant 1). However, a rather successful small-scale Jumpstart saw the development of a predictive model (random forest model, modified classification) based on historical data (oil spot prices, exchange rates, etc.). The model offered fair (70\%) accuracy and good visualization possibilities (e.g. confusion matrix, scatter plot), which were easy to understand and showed promising results. There was a mixed reception to analytics, some positive, others sceptical. The company was keen to continue and implement a production system in machine learning, but a number of problems appeared. Expectations had been raised too high, the model didn't perform so well with live production data, the historical data had been somewhat manipulated and there were data quality problems which caused the project to drag out over several months. Close cooperation in the project declined as Advectas personnel changed, and Company D staff felt a little threatened as the algorithm seemed to compete with human competences - "we can compare it [the algorithm] with human guesses, but ... [the business specialist] did not want to benchmark against the algorithm". Meanwhile the senior management had become a bit too enthusiastic: the company made a promotion video claiming that they could predict business conditions (e.g. Worldscale rate, oil prices) fourteen days ahead with $95 \%$ confidence. Relations between the two companies were turbulent for a while 
and the eventual production program offered considerably more modest results. However it is still operating and used as an input for managers' decision-making, and the company understood the potential value of predictive analytics to their business. Company D later hired their own data scientist and is clearly making a serious attempt to be a data driven company.

\section{Company E}

Company E is a large retail warehouse in Sweden, selling more than 100,000 individual product lines, and attracting customer visits from all over Scandinavia. The company wanted to predict sales for different divisions during a day, which in turn could help optimizing allocation of personnel. The financial managers were involved in the Jumpstart and supplied their own simple sales figure data, but the IT department had their own technologies and solutions and preferred not to be involved. Two of Advectas' consultants (with assistance from the sales manager) spent about three weeks developing an analysis approach based on neural networks around problems in logistics and personnel allocation. The techniques produced usable results, but were hard to explain to financial managers more accustomed to working with spreadsheets. According to consultant 4, Company E "did not understand how the algorithms worked, ... we could explain for them how time-series worked but not neural networks". Several people at Company E remained sceptical of results generated by the neural networks, and were not willing to compare them with results produced by existing manual techniques.

\section{JUMPSTART ANALYSIS}

\section{Management involvement}

Although senior management involvement is often assumed to be a pre-requisite for success, there was a varied picture in the project we studied. Company A and Company D had some direct involvement of the top management, where they also had early strategic commitments to more extensive programs. Other projects had little or no top management involvement, for instance the senior management of Company B were invited to the wrap-up event, but were not involved the Jumpstart. Nor was the involvement of top managers always beneficial: the Jumpstart for Company D was a technical success, but the senior management inappropriately raised expectations, contributing to the project running into difficulties. Though some companies showed early indications of a strategic move to enterprise-wide analytics or a data driven culture, this seemed generally beyond the scope of a Jumpstart. However, three out five Jumpstarts had middle managers with relevant expertise actively and beneficially involved: Company A (business managers), Company C (production managers), and Company E (financial managers).

\section{Analytics Experience}

Most of the client companies shared the perception that big data/advanced analytics are here to stay that they need to get started. However the starting competence level for analytics was low; ranging from the use of spreadsheets, simple data queries, and manual prediction (Company A, Company C, Company D, Company E), to no tools at all (Company B). There was little experience with mathematical modelling, machine learning algorithms or decision making based on statistical probability. An additional task for the projects was therefore building up analytics competence amongst the project members. At the kick-off events, Advectas focused on the potential of advanced analytics, in order to bring the project members on board. However there was seldom a baseline 
for existing analytical understandings (what do we know and how do we know it?), or very clear specifications for what was being analysed and why. The learning curve could be steep - sometimes too steep. Company E (more used to simple formulas in spreadsheet analysis) was offered a predictive model featuring neural networks - however this was effectively a black box that staff weren't ready to trust. A major obstacle was often the existing information infrastructure - good enough for producing financial reports but not really designed for extracting and integrating data outputs from multiple sources.

\section{The Business Problem}

A clear, limited and well-focused business problem increased the chances of success. Most of the companies had such a focused business problem requiring predictive analytics as a starting point. Company A worked on a business problem that was less precise and coupled to other marketing goals and the company's wider analytics ambitions. This encouraged project scope creep and unclear goals, and the involvement of multiple actors (for instance at workshops). It was not so clear that the business problem was solved, or that the projects led to revolutionary business advantage. The analytical models and results provided business insights not previously available, better information for decision-makers and illustrated the potential value of advanced analytics for a real business problem. There was often additional learning about the information and IT infrastructure: data quality, integration, systems, storage, platforms, algorithms etc. The Jumpstart provided a small-scale example of a new way of working where data analytics provide the foundation for decision-making, and emphasized the importance of the information infrastructure that supplies the data.

\section{People Factors}

It was noticeable at some companies that advanced analytics were challenging. The combination of lack of previous experience and a low level of trust in data, IT systems, analytics and consultants may lead to scepticism, lack of confidence, and feelings of being challenged or threatened. Three companies (A, D, E) out of five, encountered some negative reactions to advanced analytics. These companies also experienced issues related to lack of trust in the project's results. Company $\mathrm{B}$ and $\mathrm{C}$ had no obvious negative reactions. A potential explanation is that the Jumpstart in Company B and C, did not, in the short term, challenge the existing workforce. In addition, neither Company B or C had any issues with exaggerated expectations to advanced analytics.

\section{The Jumpstart Project}

The Jumpstarts generally employed the small-scale experiment model, with (not always successful) attempts at time boxing and a core project team. Several projects (A, C, D) experienced serious problems with data quality, data integration, missing data, downloading, cleaning and storing data which were time consuming and costly to fix. Prolonged data preparation also delayed modelling and analytics activities. An advanced analytics experiment is likely to use existing data in new ways, or integrate it in unfamiliar patterns, or incorporate external data. These problems compound widespread inadequacies with many companies' legacy data infrastructure. The other companies (B, E) had cleaner and simpler data available. Most Jumpstarts used a small and stable core team - typically 2-3 people from Advectas + 2-3 people from the client organization. Company D experienced several changes in the core team (partly because the data scientist skillset was in short supply and was need 
elsewhere) - this had a negative effect on the project. The Jumpstart at Company A evolved beyond the small-scale experiment - a high level of ambition, expanding requirements, multiple business problems and many people - but this was difficult to handle. Though Advectas' process model (figure 1) distinguishes between a pilot implementation and a full-scale deployment, this was not a prominent feature the projects we studied, and was not emphasised by Advectas either.

\section{Analysis summary}

Most of the projects had the following things in common. They adopted the small-scale experiment model rather than the high impact model. In principal, they were designed with a limited budget, constrained project duration, and few core project members. In this respect they were more proof of concept (show us that we can get something useful from analytics), than a revolutionary business advance. They employed advanced analytics involving some form of machine learning or statistical analysis that was well beyond the experience of the company. Middle managers with relevant business and/or IT experience were active and involved. The client company often saw the projects as an investment in competence building and they were normally satisfied when they learned more about their business, or sometimes if they learned more about their IT systems, the data they had available and new analysis possibilities. A clear business problem or hypothesis increased the chances of success. Though a pilot was always developed, a full-scale deployment was more likely to be understood as a follow-up project. The analysis is summarised in table 1:

Table 1. Analysis summary

\begin{tabular}{|c|c|c|c|c|c|}
\hline & Company A & Company B & Company C & Company D & Company E \\
\hline $\begin{array}{l}\text { Management } \\
\text { involvement }\end{array}$ & $\begin{array}{l}\text { some top } \\
\text { management } \\
\text { involvement, } \\
\text { business (middle) } \\
\text { managers involved }\end{array}$ & & $\begin{array}{l}\text { production } \\
\text { (middle) managers } \\
\text { involved }\end{array}$ & $\begin{array}{l}\text { some direct } \\
\text { involvement of top } \\
\text { management }\end{array}$ & $\begin{array}{l}\text { financial (middle) } \\
\text { managers involved }\end{array}$ \\
\hline $\begin{array}{l}\text { Analytics } \\
\text { Experience }\end{array}$ & $\begin{array}{l}\text { spreadsheets, } \\
\text { simple data queries }\end{array}$ & no tools & $\begin{array}{l}\text { spreadsheets, } \\
\text { simple data queries }\end{array}$ & $\begin{array}{l}\text { spreadsheets, } \\
\text { simple data queries }\end{array}$ & $\begin{array}{l}\text { spreadsheets, } \\
\text { simple data queries }\end{array}$ \\
\hline $\begin{array}{l}\text { The Business } \\
\text { Problem }\end{array}$ & $\begin{array}{l}\text { less precise } \\
\text { business problem }\end{array}$ & $\begin{array}{l}\text { focused business } \\
\text { problem, predictive } \\
\text { analytics }\end{array}$ & $\begin{array}{l}\text { focused business } \\
\text { problem, predictive } \\
\text { analytics }\end{array}$ & $\begin{array}{l}\text { focused business } \\
\text { problem, predictive } \\
\text { analytics }\end{array}$ & $\begin{array}{l}\text { focused business } \\
\text { problem, predictive } \\
\text { analytics }\end{array}$ \\
\hline $\begin{array}{l}\text { People and } \\
\text { Analytics }\end{array}$ & $\begin{array}{l}\text { some negative } \\
\text { reactions }\end{array}$ & $\begin{array}{l}\text { no obvious } \\
\text { negative reactions }\end{array}$ & $\begin{array}{l}\text { no obvious } \\
\text { negative reactions }\end{array}$ & $\begin{array}{l}\text { some negative } \\
\text { reactions }\end{array}$ & $\begin{array}{l}\text { encountered some } \\
\text { negative reactions }\end{array}$ \\
\hline $\begin{array}{l}\text { The } \\
\text { Jumpstart } \\
\text { Project: }\end{array}$ & $\begin{array}{l}\text { serious data quality } \\
\text { problems, many } \\
\text { people involved, } \\
\text { deployment follow- } \\
\text { up }\end{array}$ & $\begin{array}{l}\text { small and stable } \\
\text { core team, small- } \\
\text { scale experiment }\end{array}$ & $\begin{array}{l}\text { serious data quality } \\
\text { problems, small } \\
\text { and stable core } \\
\text { team, small-scale } \\
\text { experiment }\end{array}$ & $\begin{array}{l}\text { serious data quality } \\
\text { problems, several } \\
\text { changes in the core } \\
\text { team, small-scale } \\
\text { experiment }\end{array}$ & $\begin{array}{l}\text { small and stable } \\
\text { core team, small- } \\
\text { scale experiment }\end{array}$ \\
\hline
\end{tabular}




\section{THE ADVANCED ANALYTICS JUMPSTART: DEFINTION, PROCESS MODEL, BEST PRACTICES}

\section{Jumpstart definition}

As a result of this investigation we understand an AA Jumpstart to be an introductory AA project in a client company inexperienced in analytics, led by a specialist consultancy. The project is designed as a small-scale experiment, with low costs and low risks for the client and with significant interaction between consultants and company staff. Jumpstarts are focused at delivering business value, but also offer various knowledge transfer and catalyst outcomes. Above all they should stimulate further interest in the client company, inspiring new initiatives and functioning as proof of concept - the client learns enough about the value of AA to wish to continue. The consultancy hopes to develop a relationship with the client and win further business.

\section{Jumpstart process model}

Figure 3 offers a modified and amplified version of Advectas' Jumpstart process (figure 1) that is in harmony with what we observed in the case examples. Major phases are specified together with deliverables, such that it could serve as a project management tool for a consultancy managing a Jumpstart.

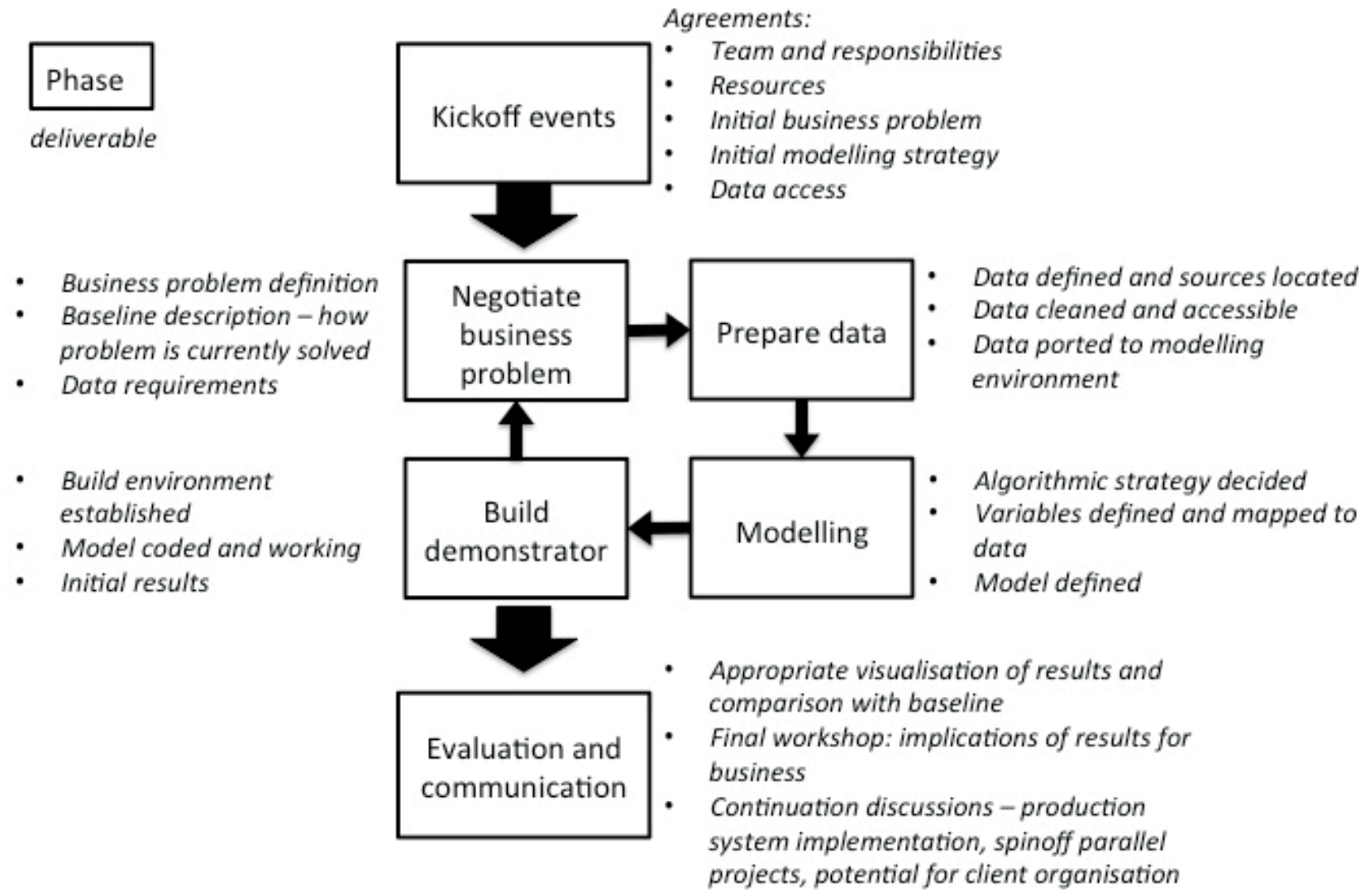

Figure 3. Jumpstart process 


\section{Jumpstart best practices}

This section takes the form of normative advice for consultants running Jumpstarts derived from the theoretical and empirical analysis.

\section{Management Involvement}

Though management should be involved at various levels, the role of middle management in a Jumpstart is more critical to its success.

- Ensure that top management are supportive (they're not necessarily directly involved). Top management may need to authorize resources for the Jumpstart and show an interest, but the scale of the projects are usually too small for them to be directly involved.

- Actively involve middle management owning the business problem. Local managers are crucial to the success of the Jumpstart, supplying invaluable knowledge of business needs and data configurations, driving the project, solving practical problems and shortcutting bottlenecks. They improve their analytics competence (both business-oriented and technicaloriented) in the project, and take responsibility for knowledge transfer and realistic feedback to IT and business managers not directly involved.

- Negotiate realistic expectations for project outcomes with management. Advanced analytics are currently surrounded by a great deal of hype, and managers can easily be swept away by exaggerated claims and expectations. Small-scale experiments with limited resources are unlikely to change the future of a company, but they can provide valuable insights into specific business problems, and demonstrate the potential value of analytics. Setting explicit and realistic goals and targets (and achieving them) is better than letting expectations run and then disappointing the personnel.

\section{Analytics Experience}

Many Jumpstart organizations have a low level of analytical sophistication, which has consequences for the ambition-level and conduct of the Jumpstart.

- Understand the company's analytics maturity and focus on explanation and education (particularly at kick-off and wrap-up events). Low levels of analytics experience mean that most Jumpstarts must focus on knowledge transfer and competence development in order to succeed. An early Jumpstart task is to assess the starting competence level at the client company, and provide the necessary explanation and education at kick-off to bring project members on board. The wrap-up event should focus on lessons learnt, and highlight advances in analytics understanding.

- Match the analytics complexity of the Jumpstart to the maturity of the company. Many companies have little experience of analytics beyond the use of spreadsheets and simple dashboards, and the first steps are challenging. Here an algorithmic approach that can be explained in relatively straightforward terms can increase both the chances of acceptance, and trust in the results. Complex techniques (neural networks and deep learning) should normally be saved for more experienced organizations. 


\section{Business Problem}

A focused business problem utilizing predictive analytics was advantageous.

- Choose a limited business problem focusing on predictive analytics. Descriptive analytics typically require a data warehouse solution (beyond the scope of a Jumpstart), and prescriptive analytics assumes that predictive insights are already available and ready to be automated. A limited business problem involving predictive analytics is the preferred choice.

- Formulate the business problem as clearly as possible. Small scale projects require a limited scope and clarity of purpose. A clearly formulated problem reduces the risk of confusion, lack of focus and delays as the project progresses.

- Describe the existing ways of addressing the problem (the baseline). The perceived success of the project is normally dependent on being able to demonstrate an improvement, or solution to the problem. Briefly documenting the existing way of tackling the problem establishes a baseline, though this can be sensitive (since it is sometimes an area of concern in the company). The eventual Jumpstart results should show at least the potential for an improvement to the baseline.

People Factors

Jumpstarts need to deal with negative reactions as well as enthusiastic ones.

- Address expectations and anxiety. The people directly or indirectly involved in a Jumpstart may be inexperienced with advanced analytics. This can lead to two problems: exaggerated expectations or negative reactions. Negative reactions may include scepticism, distrust, and feeling challenged or threatened. Some may even feel that their jobs are threatened. Jumpstart teams should recognize and, as far as possible, address these concerns. Most advanced analytics tools provide better (but not infallible) support for decision makers they do not replace the decision maker.

- Build trust by patiently explaining issues and techniques, and by showing that the analytics results are reliable and dependable. The data foundation for a Jumpstart is often incomplete and faulty, the computational techniques involved are difficult to understand, and the models developed are often black boxes. This means that trust in the project's results can be very low. Jumpstarts need to build trust by explaining the issues involved and how they are tackled, by providing non-mathematical analogies for the techniques used, and by showing a consistent stream of improving results.

\section{Jumpstart Project}

The four previous enablers (management involvement, analytics experience, business problem, and people factors) influence, but do not determine the conduct of the Jumpstart project.

- Design the project as a small-scale experiment, with a constrained business problem, a small (carefully chosen) core project team, and time boxed execution. Projects are more manageable (and therefore effective) when organized in this way. The experimental form allows the client organization to assess the potential value of analytics; the constrained 
business problem ensures that the project objectives cannot multiply out of control. A core project team might include from the consultancy side: project manager, data scientist, data engineer; from the client side: business specialist (problem owner) with in-depth knowledge of the problem and data, IT person with knowledge of IT feeder systems, manager with the authority to problem solve and organize resources. The project is more effective if the team remains intact and this is easier to achieve if there is time boxing (1-2 months), which also helps to avoid creep in the project scope, and ensures that there are timely results.

- Address data quality early. Since most companies data infrastructure is not organized with the purpose of providing data for advanced analytics, acquiring and preparing the data can be a difficult and costly. A Jumpstart should negotiate how to proceed in this case, and make sure the problem is explicit and budgeted for.

- Explain the results carefully in terms that the client can understand, and demonstrate the progress that has been made. It's not the client's responsibility to appreciate the power of advanced analytics, it's the Jumpstart project's responsibility to explain its results in terms that business and IT people can understand. The consultancy specialists need to take the lead, and a variety of appropriate visualizations and careful explanations will help. A wrapup meeting should also specify the progress that has been made on the baseline, in terms of business insights, better information for decision makers and analytics learning.

\section{DISCUSSION}

The literature we studied deals primarily with business intelligence and big data projects conducted internally in organisations, and here we contrast this with our investigation of AAJumpstarts. Though involvement from senior management is usually understood to be critical (Brown \& Gottlieb, 2016; Groves et al., 2013; Mazzei et al., 2016), we found that the active involvement of middle management was more important in ensuring Jumpstart success. They provided invaluable business knowledge, smoothed the path of the project in the face of anxieties, were active learners and the initiators of future steps. C-suite executives sometimes authorized resources and provided symbolic backing. All our case studies chose a business problem that explored data using predictive analytics, rather than descriptive or prescriptive analytics. We surmise that descriptive analytics provide too little that is innovative, whereas prescriptive analytics are beyond the reach of inexperienced companies. Jumpstarts tackled a variety of different business problems in different business areas, and it was not really significant which area was chosen - more important was precise focus and limited scope. Advanced analytics can be used in many situations, but there was less involvement with typical big data concerns. Companies were still mainly reliant on their own internal data, though there was some use of external data (e.g. social media), and unstructured data. Jumpstart consultancies worked with primarily inexperienced companies - typically at the aspirational level of analytics maturity (LaValle et al., 2010). This reflects a situation, even in technologically advanced countries, where a few large tech companies are analytically mature, but the majority of organizations have to play catch up from a low base. Both business-oriented and technically oriented competences were in need of development. The consultants' role was to integrate some data science, data engineering and Jumpstart expertise so that the client company could compensate for some missing expertise - providing a kind of bridging role as suggested by Ohlsson (2017). The open style of exploratory data analysis favoured by technical AA researchers was not very successful in Jumpstarts - a focused problem or hypothesis was preferred, and the consultancy company had a role to play in helping with inexperience in problem selection 
(Wixon et al., 2013). We found evidence that the integration of diverse skill sets was difficult and important. We found both (positive) enthusiasm and (negative) lack of trust for analytics (not only amongst management), but the scale of a Jumpstart is usually too small to be threatening to senior managers. On some occasions AA showed the potential to automate the functions of operational staff, and given the long history of automation related redundancy with IT, this might be a powerful people factor in the future. Jumpstarts favoured the small-scale experiment project model over the high impact model. There is no particular reason why a consultancy company should not be involved in a major organization change program, for instance the enterprise-wide move to a data driven culture. However the small-scale experiment model is a low risk alternative with good potential for small quick wins. Though project process is sometimes based on CRISP-DM, sometimes on software development (Larson \& Chang, 2016), AA Jumpstarts show sufficiently distinctive characteristics to warrant independent consideration. A final observation is that it is unclear in the literature what the step after Jumpstart might be; where should an organization on the AA journey go next?

\section{CONCLUSIONS}

In this article we investigated the experiences of five different Swedish organizations starting their journey towards in advanced analytics through the vehicle of a Jumpstart - a consultantdriven introduction project. The common factor in the projects was the consultancy company, which provided specialist expertise in advanced analytics not otherwise available to the companies - we took primarily the consultant perspective in this study. There is no strong theory base in early stage AA introduction, or the role of consultancy companies in these first steps. We conducted a qualitative investigation of the Advectas experience through interviews, document study and workshops, using content analysis as the primary analysis of the five cases. We concentrated on five theory-derived enablers: management involvement, analytics experience, business problem, people factors, and the Jumpstart project itself. The contribution is a definition, a process model, and set of best practices derived jointly from existing theory and the empirical analysis, which are intended to be of value to practitioners in the field, and to inform researchers.

We can point to the following implications for practitioners. It is expected that advanced analytics are here to stay in some capacity in all but the smallest organizations, so the majority of companies in advanced technological societies will make this transition, if they have not already started. Specialist data analytics consultancies can play an important role in taking the first steps, with a range of skills including problem selection, data science competence, data management, model construction, result visualization, skillset integration, Jumpstart management, analytics education and benefits management. Jumpstart is effective in the sense that a small number of consultancy firms with specialist analytics expertise, can demonstrate business value and increase trust in AA in a large number of organisations in a relatively short time. Consultants provide the missing expertise for an initial AA project. They may also jumpstart the organisation along multiple dimensions, rather than providing technical insight alone. As a consequence they have an important role in the diffusion and adoption of these analysis technologies, which are also unavoidable in the analysis of big data. The first steps may not necessarily result in revolutionary business advantage, but a range of benefits can be expected. However success is not guaranteed and careful attention should be paid to the practices outlined above. Special attention might be paid to two factors. Data quality and accessibility should be addressed early - this is a recurring problem with many organizations and impacts both the effectiveness of the project and trust in its results. Expectations and anxiety should also be addressed 
- this is a major factor in the future impact of a successful project. A technically successful project has little impact if its results are not trusted and used. Researchers may care to complement the extensive research on algorithmic techniques for problem solving with more integrative research that focuses on how to make existing techniques accessible and understandable for practitioners, how to couple them in to real-life data and legacy systems, and practice-based research into how to move organizations through early analytics experience towards data-driven decision-making embedded in organisational culture.

The Swedish experience, although not expected to be untypical of advanced technological societies, need to be corroborated in other similar societal contexts, and this research is also limited by focusing on the experience of a single consultancy company. Though other companies run similar Kickstart and Jumpstart programs it is not known how representative the Advectas cases are. We worked with loose definitions of AA project success and would like to develop formal definitions and if possible metrics for this. We also intend to investigate how organizations work with establishing data-driven decision-making practice, and the role of specialized data analytics consultancies in this progression.

\section{ACKNOWLEDGEMENT}

The work is partially supported by the Knoweldge Foundations of Sweden.

\section{REFERENCES}

Barton, D., \& Court, D. (2012). Making Advanced Analytics Work For You. Harvard Business Review, 90(10), 78-83.

Benbasat, I., Goldstein, D. K., \& Mead, M. (1987). The Case Research Strategy in Studies of Information Systems. MIS Quarterly, 11(3), 369-386.

Berelson, B. (1952). Content Analysis In Communicative Research. New York: Free Press.

Brown, B., \& Gottlieb, J. (2016). The need to lead in data and analytics. Retrieved from McKinsey \& Company:

Carroll, J. M., \& Swatman, P. A. (2000). Structured-case: a methodological framework for building theory in information systems research. European Journal of Information Systems, 9(4), 235-242.

Chen, H., Chiang, R. H., \& Storey, V. C. (2012). Business Intelligence and Analytics: From Big Data to Big Impact. MIS Quarterly, 36(4), 1165-1188.

Davenport, T. H. (2013). Analytics 3.0. Harvard Business Review (December).

Davenport, T. H., Harris, J. G., \& Morison, R. (2010). Build an analytical culture Analytics at Work: Smarter Decisions, Better Results: Harvard Business Press.

Davenport, T. H., \& Patil, D. J. (2012). Data Scientist: The Sexiest Job of the 21st Century. Harvard Business Review, 90(10), 70-76.

Eisenhardt, K. M., \& Graebner, M. E. (2007). Theory building from cases: Opportunities and challenges. Academy of management journal, 50(1), 25-32.

Franks, B. (2012). To Succeed with Big Data, Start Small. Harvard Business Review Digital Article.

Gartner IT Glossary. (2017). http://www.gartner.com/it-glossary/advanced-analytics 
Groves, P., Kayyali, B., Knott, D., \& Van Kuiken, S. (2013). The 'big data' revolution in healthcare. Retrieved from McKinsey Quarterly:

He, W., Zha, S., \& Li, L. (2013). Social media competitive analysis and text mining: A case study in the pizza industry. International Journal of Information Management, 33(3), 464-472.

Henke, N., Bughin, J., \& Chui, M. (2016). Most Industries Are Nowhere Close to Realizing the Potential of Analytics. Harvard Business Review Web Article.

Krippendorff, K. (2004). Content Analysis. Thousand Oaks: Sage.

Kumar, V. D., \& Alencar, P. (2016). Software engineering for big data projects: Domains, methodologies and gaps. Paper presented at the 2016 IEEE International Conference on Big Data (Big Data).

Lahrmann, G., Marx, F., Winter, R., \& Wortmann, F. (2011). Business intelligence maturity: Development and evaluation of a theoretical model. Paper presented at the Proceedings of the 44th Hawaii International Conference on System Sciences.

Larson, D., \& Chang, V. (2016). A review and future direction of agile, business intelligence, analytics and data science. International Journal of Information Management, 36(5), 700-710. doi: http://dx.doi. org/10.1016/j.ijinfomgt.2016.04.013

LaValle, S., Hopkins, M. S., Lesser, E., Shockley, R., \& Kruschwitz, N. (2010). Analytics: The New Path to Value. Retrieved from MIT Sloan Management Review:

LaValle, S., Lesser, E., Shockley, R., Hopkins, M. S., \& Kruschwitz, N. (2011). Big Data, Analytics and the Path From Insights to Value. MIT Sloan Management Review, 52(2), 21-32.

Marchand, D. A., \& Peppard, J. (2013). Why IT Fumbles Analytics. Harvard Business Review, 91(1-2), 104112.

Mazzei, C., McShea, C., \& Oakley, D. (2016). How CEOs Can Keep Their Analytics Programs from Being a Waste of Time. Harvard Business Review Web Article.

Miele, S., \& Shockley, R. (2013). Analytics: The real-world use of big data. Retrieved from IBM Institute for Business Value, Said Business School:

Ohlsson, F. (2017). Building a global culture of analytics. Beslutsstödsdagen 2017, Stockholm.

Rose, J., \& Lennerholt, C. (2017). Low Cost Text Mining as a Strategy For Qualitative Research. Electronic Journal of Business Research Methods, 15(1), 2-16. http://issuu.com/academic-conferences.org/docs/ ejbrm-volume15-issue1-article447?mode=a $\_$

Saltz, J. S. (2015). The need for new processes, methodologies and tools to support big data teams and improve big data project effectiveness. Paper presented at the 2015 IEEE International Conference on Big Data (Big Data).

Shah, S., Horne, A., \& Capellá, J. (2012). Good data won’t guarantee good decisions. Harvard Business Review, 90(4).

Turban, E., Sharda, R., Delen, D., Aronson, J. E., Lian, T.-P., \& King, D. (2015). Business intelligence and analytics : systems for decision support. Harlow, Essex: Pearson Education.

Watson, H. J. (2016). Creating a Fact-Based Decision-Making Culture. Business Intelligence Journal, 21(2), $5-9$.

Vidgen, R., Shaw, S., \& Grant, D. B. (2017). Management challenges in creating value from business analytics. European Journal of Operational Research, 261(2), 626-639.

Wilkinson, S. (1997). Focus group research. In D. Silverman (Ed.), Qualitative Research: Theory, Method and Practice (pp. 177-199). London: Sage. 
Wirth, R., \& Hipp, J. (2000). CRISP-DM: Towards a standard process model for data mining. Paper presented at the Proceedings of the 4th International Conference on the Practical Applications of Knowledge Discovery and Data Mining.

Wixon, B. H., Yen, B., \& Relic, M. (2013). Maximizing Value from Business Analytics. MIS Executive Quarterly, 12(2), 111-123.

Yin, R. K. (2013). Case study research: Design and methods (4th ed.). Thousand Oaks, California: Sage Publications. 
Appendix A. Data collection

\begin{tabular}{ccccc}
\hline Reference & Date & Organisation & Type & Purpose \\
\hline 1 & March 1 2016 & Company A + Advectas & On site & $\begin{array}{c}\text { Introduction to analytics project at } \\
\text { Company A }\end{array}$ \\
\hline 2 & May 11 2016 & Company A (IT/IS) & Phone & Introduction to analytics project \\
\hline 3 & May 31 2016 & $\begin{array}{c}\text { Company A } \\
\text { (Head of analytics vision) }\end{array}$ & On site & In depth vision of analytics project \\
\hline 4 & May 31 2016 & Company A (IT) & On site & IT's role in the analytics project \\
\hline 5 & May 31 2016 & Company A (Marketing) & On site & Business' role in the analytics project \\
\hline 6 & October 28 2016 & Advectas & On site & Initial planning of case studies \\
\hline 7 & November 22 2016 & Advectas & Phone & Overview of case studies \\
\hline 9 & December 16 2016 & Advectas Consultant 1 & Skype & Company D case \\
\hline 10 & December 16 2016 & Advectas Consultant 1 & Skype & Company B case \\
\hline 11 & January 10 2017 & Advectas Consultant 3 & Skype & Company A case \\
\hline 12 & January 20 2017 & Advectas Consultant 4 & Skype & Company E case
\end{tabular}

Appendix B. Coding example

\begin{tabular}{|c|c|c|c|c|c|c|}
\hline \multirow{2}{*}{ Category } & \multirow{2}{*}{ Feature } & \multicolumn{5}{|c|}{ Company Company Company Company Company } \\
\hline & & $\mathbf{A}$ & B & $\mathbf{C}$ & D & $\mathbf{E}$ \\
\hline T1: Management & IT management involvement & $\mathrm{x}$ & $\mathrm{x}$ & & & \\
\hline T1: Management & financial managers involved & & & & & $\mathrm{x}$ \\
\hline T1: Management & top managers involved & & & $\mathrm{x}$ & $\mathrm{x}$ & \\
\hline T1: Management & $\begin{array}{l}\text { ambition to be data driven/ } \\
\text { organisational change }\end{array}$ & $\mathrm{x}$ & & & $\mathrm{x}$ & \\
\hline T1: Management & production managers involved & & & $\mathrm{x}$ & & \\
\hline T2: Analytics & $\begin{array}{l}\text { little experience/ } \\
\text { immature in analytics }\end{array}$ & & $\mathrm{x}$ & & $\mathrm{x}$ & $\mathrm{x}$ \\
\hline T2: Analytics & $\begin{array}{l}\text { experience with business } \\
\text { intelligence }\end{array}$ & $\mathrm{x}$ & & & & \\
\hline T2: Analytics & little trust for analytics & $\mathrm{x}$ & & & & \\
\hline T3: Business problem & $\begin{array}{l}\text { results in new decision/ } \\
\text { change/new hiring }\end{array}$ & & $\mathrm{x}$ & & $\mathrm{x}$ & \\
\hline T3: Business problem & driven by business & $\mathrm{x}$ & & $\mathrm{x}$ & & $\mathrm{x}$ \\
\hline T3: Business problem & sales & & & & & $\mathrm{x}$ \\
\hline T3: Business problem & $\begin{array}{l}\text { clear business problem or } \\
\text { hypothesis }\end{array}$ & & $\mathrm{x}$ & $\mathrm{x}$ & $\mathrm{x}$ & \\
\hline T3: Business problem & marketing & $\mathrm{x}$ & & & & \\
\hline
\end{tabular}

